\title{
Effect of Epoxomicin on 3D Neurospheres
}

Salama M. , Khaled Youssef, Mohamed Elhosseny, Bahaaeldin Awad, LotfyA. , El-gamalM., , Sheashaa H. , , Sobh $\mathrm{M}^{3,4}$

1-Toxicology Department, Faculty of Medicine, Mansoura University.

2-Medical Experimental Research Center (MERC), Faculty of Medicine, Mansoura University, Mansoura, Egypt.

3-Faculty of Medicine, Mansoura University, Mansoura, Egypt

4-Nephrology Department, Urology \& Nephrology Center, Mansoura University, Egypt.

\section{Summary}

In the present study we verified the possible Developmental neurotoxicity (DNT) of Epoxomicin. We found that it affects differentiation and viability of Neuropsheres these effects were positively correlated to doses and progress of time.

\section{Introduction}

DNT entails the toxic effects imparted by various chemicals on brain during the early childhood period. Most of toxicants can not be identified due the limitations and defective nature of predictive toxicology models used[1].

A novel alternative method that can overcome most of the limitations is the use of 3D Neurospheres system [2]. This in-vitro system can recapitulate most of the use of $3 \mathrm{D}$ Neurospheres system [2]. This in-vitro system can recapitulate most of the
changes during the period of brain development making it an ideal model for predicting neurotoxic effects.

Epoxomicin which is a naturally occurring selective proteasome inhibitor with anti-inflammatory and anti-tumor activity, selectively,

and irreversibly inhibits proteasome activity . [3]

\section{Methodology}

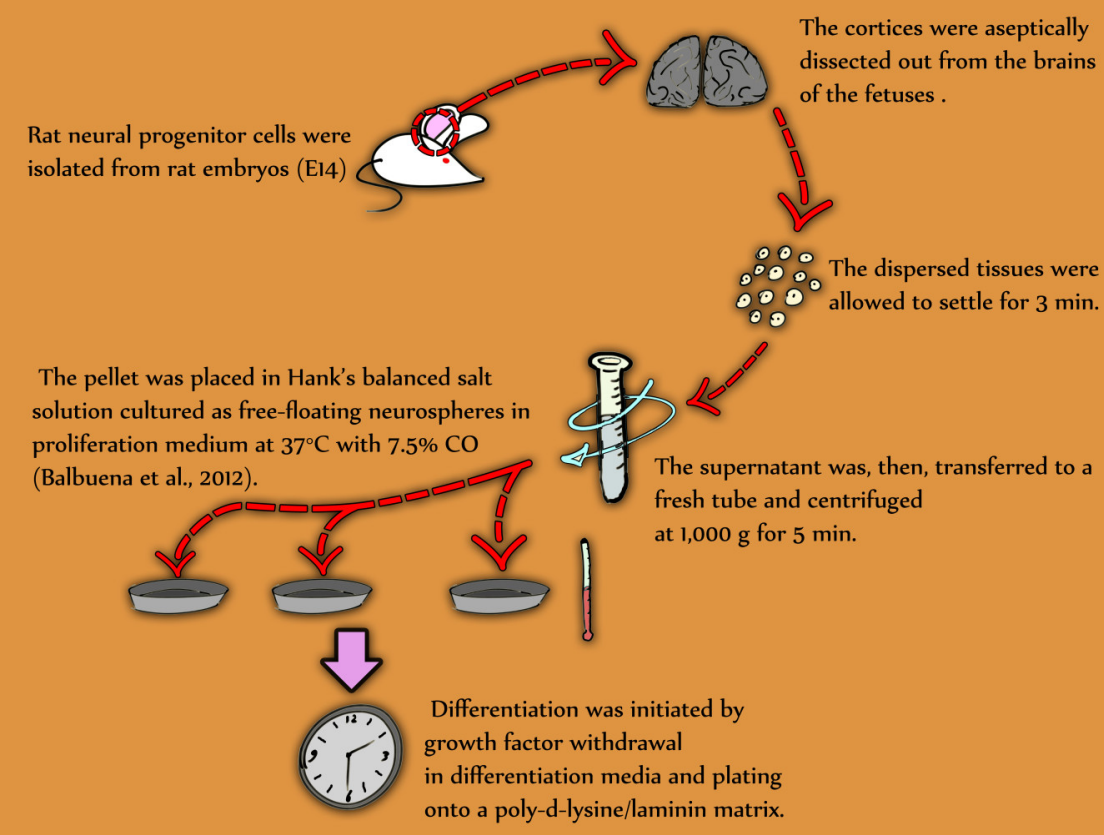

Chemical Exposure. For proliferation analysis, neurosphereswere treated for 2 weeks with Epoxomicin $(1,10 \mu \mathrm{M})$ in proliferation medium.

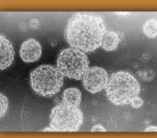

Proliferation analysis. spheres were cultured in proliferation medium. After $0,3,7,10$ and 14 days, sphere size was determined by software analyses (Cell Profiler, version 2.l; Broad Institute, freely downloaded from http://www.cellprofiler.org). Diameter of each neurospherewas measured and
exported to Excel file further to statistical analysis. Fig.(1)

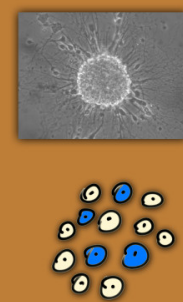

Differentiation analysis. following initiation of differentiation as described previously, images of plated neurosphereswere evaluated regarding distinet neuronal morphology with fasciculaton of nedrits that radiate from the central aggregation of neuronal perikary.

Viability analysis. trypsin-EDTA solution were added to neurospheres for 3 min to dissociate them into single cells suspension, then viability evaluated by the Trypan Blue exclusion test. Fig.(2).

\section{Results}

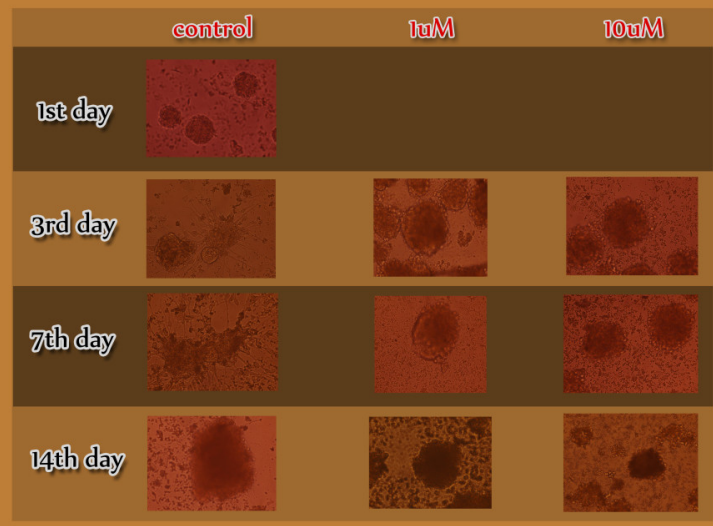

Table ( 1 ): Differentiation analysis of Epoxomicin treated groups $\mathrm{X}_{20}$

Following initiation of differentiation, neurospheres in the control group showed distinct neuronal morphology with fasciculation of neuritis. However, Epoximicin treated neurospheres failed to show this neurogenic differentiation.

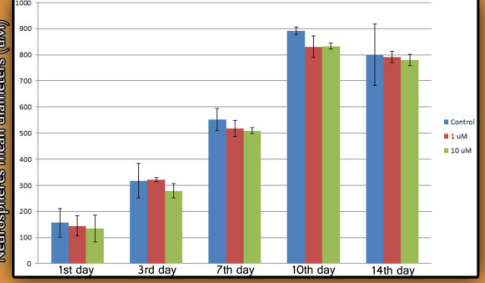

Fig.(1): Bar chart showing the mean of the diameters of neurospheres through the period of two weeks.

Proliferation of cells exposed to Epoximicin was not significantly affected

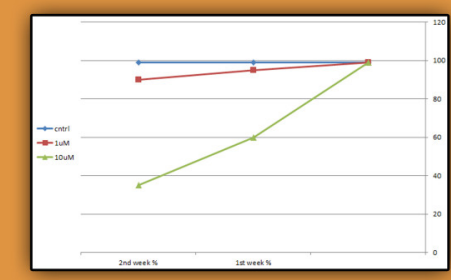

Fig.(2): Linear Chart \% of viable cells in the two treated groups compared to control

\section{Conclusion}

The findings of the present study confirm the negative impact of Epoxomicinon differentiation and viability of Neural Progenitor cells (NPCS) which reflects possible developmental disorders that he DNT of E on proliferation of $3 \mathrm{D}$ neurospheres.

\section{References}

1.Ostrea EM Ir, Reyes A, Villanueva LyE, PacificoR, Benitez B, Ramos E, Bernardo RC,

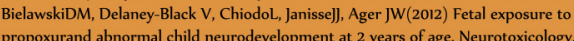
33(4):669-675.

2.Moors M, RockelTD, Abel l, Clinc EE, Gassmannk, Schreciber T, Schuwald, WeinmannN, FritscheE(2009) Human neurospheresass three-dimensional cellular system

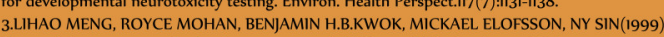
Epoxomicin, a potent and selective proteasome inhibitor, exhibits in vivo anti-inflammatory activity 